\title{
Bilateral Tension Pneumothoraces Leading to Cardiac Arrest after Coronary Artery Bypass Surgery
}

\author{
Anthony Alozie ${ }^{1}$ Bernd Westphal ${ }^{1}$ Can Yerebakan ${ }^{1}$ Gustav Steinhoff ${ }^{1}$ \\ ${ }^{1}$ Department of Cardiac Surgery, University of Rostock Medical \\ Faculty, University Heart Center Rostock, Rostock, Germany \\ Address for correspondence Dr. Anthony Alozie, M.D., Department of \\ Cardiac Surgery, University of Rostock Medical Faculty, University \\ Heart Center Rostock, Schillingallee 35, 18057 Rostock, Germany \\ Thorac Cardiovasc Surg 2012;60(S2):e6-e8. \\ (e-mail: uju77de@yahoo.de).
}

\begin{abstract}
Bilateral pneumothoraces are a very rare event. In clinical settings, inadvertent incursion into the pleural space resulting from diagnostic or therapeutic medical interventions such as bilateral venipunctures or damage to the lung parenchyma due to high pressure ventilation may be causative. Bilateral pneumothoraces postcardiac surgery are rarely reported. We present the case of bilateral tension pneumothoraces leading up to cardiorespiratory arrest in a 57-year-old male, weighing $130 \mathrm{~kg}$, who underwent without any complications a coronary artery bypass surgery. Thoracic chest tubes (retrosternal

\section{Keywords}

- pneumothorax

- chest

- postoperative care and intrapericardial) and a left pleural tube were removed 24 hours prior to the incident. Diffuse sternal pain sensation accompanied by slow progressive respiratory distress and confusion shortly before the incident were all developed over a period of 12 hours. A prompt cardiopulmonary resuscitation and bilateral chest tube insertion had prevented a fatality in the patient.
\end{abstract}

Tension pneumothorax is generally regarded as an immediate life-threatening condition. The involvement of both hemithoraces is rare and may occur in the postoperative setting as a complication of sternotomy in a heart surgery. Failure to recognize the early signs and symptoms of tension pneumothorax as well as providing prompt treatment using needle decompression or thoracostomy tube may result in a collapse of the cardiorespiratory function. In bilateral tension pneumothoraces without having rapid and optimal vent for decompression, the impaired ventilation and increased intrathoracic pressure which potentially resulting in reduced venous return to the heart could both contribute toward a rapid cardiorespiratory collapse. ${ }^{1}$

\section{Case Report}

A 57-year-old male who has smoked for 30 years presented with effort-associated retrosternal pressure and pain sensation. In his medical history, there were notable multiple

percutaneous coronary interventions and stent grafting on both the left anterior descending (LAD) and right coronary arteries (RCA), respectively. Hypertension, diabetes mellitus, and obesity were also noted. Coronary angiography demonstrated a three-vessel disease and coronary artery bypass graft surgery was performed through a median sternotomy. During sternotomy, the left pleural cavity was visibly entered by accident. At the end of the surgery, a left pleural chest tube in addition to the retrosternal and intrapericardial tubes were inserted to ensure optimal drainage of the mediastinum and pleura. The surgery went without any complications and the patient was transferred to the normal ward on the first postoperative day from the intensive care unit (ICU). Chest tubes were removed routinely on the evening of the first postoperative day. The chest X-ray showed no abnormalities (-Fig. 1A).

After 12 hours, he reported a mild diffuse thoracal pain sensation and dyspnea which responded temporarily to peripheral analgetics and oxygen insufflation. Oxygen

(c) 2012 Georg Thieme Verlag KG Stuttgart · New York
DOI http://dx.doi.org/ 10.1055/s-0031-1295577. ISSN 2194-7635. 


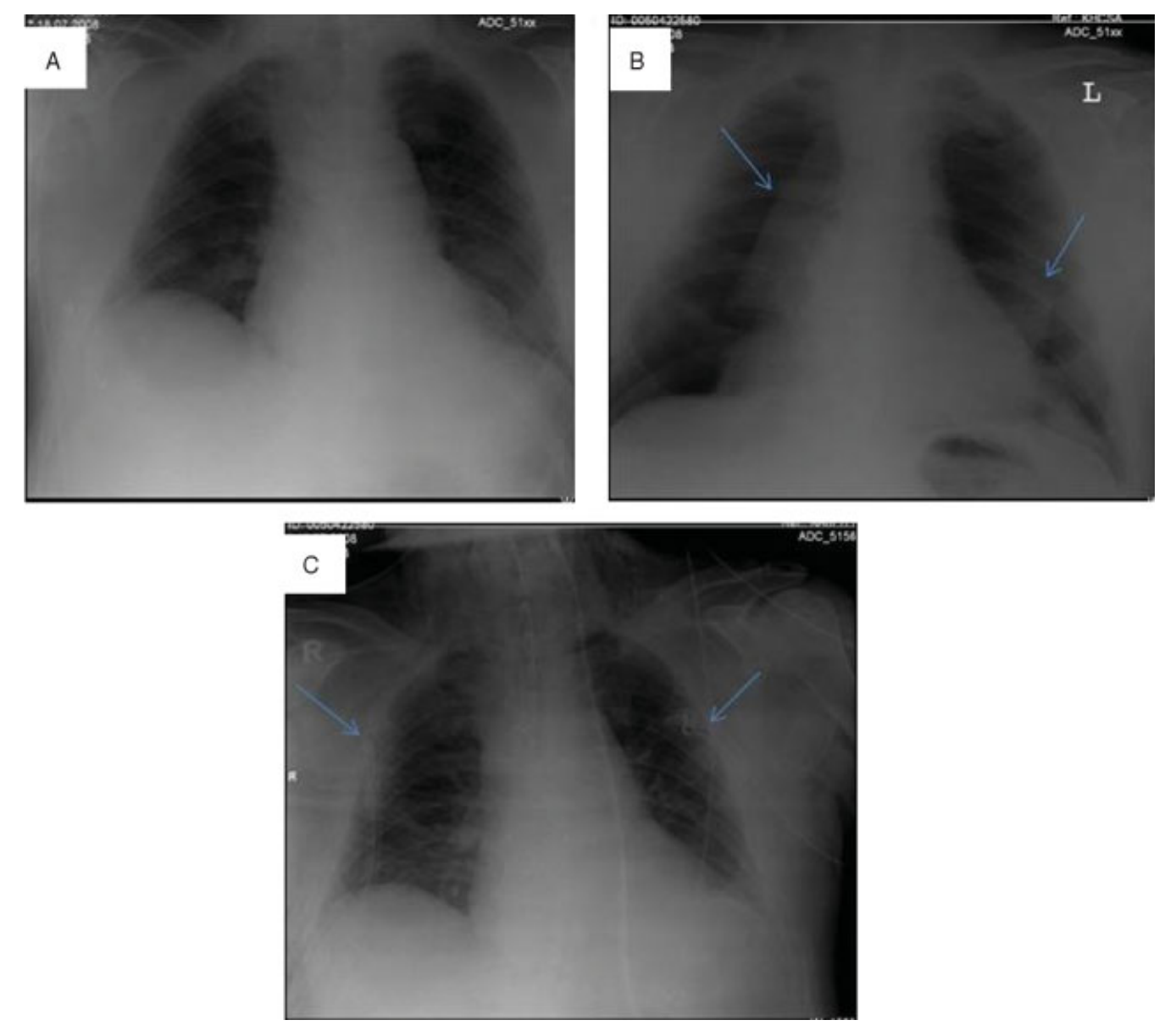

Figure 1 Chest X-ray showing a normal X-ray 2 hours after chest tube removal (A). Total collapse of both lungs (B). Bilateral tube thoracostomy led to restoration of cardiorespiratory function (C).

saturation on $2 \mathrm{~L} / \mathrm{min}$ oxygen flow remained normal with $97 \%$. Four hours thereafter, the patient again reported diffuse thoracic pains. This time, the peripheral analgetics did not show adequate pain relief and thus piritramide was prescribed, which subsequently relieved him of his pains with Spo2 returning to values between 96 and 97\%.After 6 hours, he developed tachypnea (20/min) and signs of restlessness. While the patient was awake with no symptoms and fully responsive, a repeated chest X-ray was ordered along with repetitive chest percussion and auscultation to reevaluate his cardiopulmonary function. Declined breath sounds over the left hemithorax suggested a pneumothorax and needle decompression was indicated. Throughout these procedures, his Spo 2 decreased to values between 90 and $92 \%$ coupled with development of marked respiratory distress, cyanosis, confusion, distended jugular veins, hypotonia, and then cardiorespiratory arrest. Cardiopulmonary resuscitation was immediately initiated with intubation of the patient. This however, despite presumed correct placement of the tube failed to yield any favorable results. Bilateral absence of breath sounds upon intubation further supported the tentative diagnosis of bilateral pneumothoraces, which was later

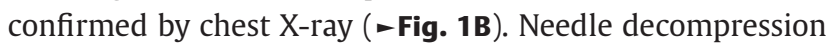
on the left-sided pneumothorax at first led to signs of improvement. Further amelioration of the patient's condition was achieved with bilateral chest tube placement with reexpansion of both lungs (- Fig. 1C)

He was later put under anesthesia and transferred to the ICU. His gas exchange improved and respiratory support in the ICU was discontinued after 8 hours. Air leakage from both chest tubes persisted for 2 to 3 days, respectively, where they were later removed upon cessation. We recommended a computer tomography scan (CT) assessment for exclusion of occult lung pathologies upon discharge on the 10th day postoperative into a rehabilitation center.

\section{Discussion}

Pneumothorax results from injuries to the trachea, lung parenchyma, or the chest wall. In cardiac surgical patients, bilateral pneumothoraces may potentially occur if both pleura spaces are inadvertently being entered at the time of sternotomy. This may result in a common pleural space (iatrogenic buffalo chest) which subsequently places the patient in a unique danger when air enters one hemithorax. $^{2-4}$

The diagnosis of large pneumothoraces is normally made through physical examination. Chest X-ray, CT- scan, and thoracoscopic examination are well-established diagnostic methods in confirming both large and small pneumothoraces 
if needed. Small asymptomatic pneumothoraces may be treated in a conservative manner. However, large or tension pneumothoraces may pose a danger to patients cardiorespiratory function which should be timely identified and be treated as an emergency. ${ }^{5}$

In lieu of a well-defined disease state, there is probably a subset of robust patients whose symptoms progress much more slowly due to their ability to compensate through other mechanisms. In this subclass of patients, the classic symptoms may be absent or are developing much more slowly, leaving only none-specific findings such as pain, dyspnea, and tachypnea. Early indication for chest X-ray, CT- scans, and thoracic ultrasound should be timely put into the consideration to rule out other causes of such symptoms to prevent unnecessary endangerment of the patient. This should in particular be considered when patients after undergoing a sternotomy, present with slow progressive thoracic pains and respiratory distress. ${ }^{6,7}$

Pneumothoraces occurring in patients postcardiac surgery is not rare, whereas bilateral tension pneumothoraces after heart surgery has rarely been reported ${ }^{3,8}$

Pneumothorax arises when the negative intrapleural pressure is disrupted. Treatment of a tension pneumothorax after the diagnosis has been established involving oxygen application and immediate decompression. Total collapse of both lungs leaves the patient, though incubated, in a situation whereby the patient has insufficient ventilation, which leaves rapid bilateral decompression as the only life-saving remedy.

In our patient, a credible pathogenesis may be the presence of interpleural communication created during median sternotomy. It seems likely that this had led to the formation of single pleura, a situation known as "Buffalo chest." This has been coined for the condition on the basis that buffalos or bison have a single pleural cavity. Communications between both pleural cavities may be congenital, iatrogenic especially postheart surgery, laparoscopic or traumatic in etiology, and have been reported in the related published medical journal and case report. ${ }^{2-4}$ The result of communicating pleural cavities would be bilateral pneumothoraces if air continuously gains entrance into one pleural space.

Is it possible that the iatrogenic entrance to the pleura initially communicated with the atmospheric air via surgical entrances for the chest tubes? These are traditionally placed via skin incision in the epigastrium. This may potentially have provided a one-way valve after the removal of the chest tubes. The gradual air entrance with progressive intrapleural pressure could have raised a small iatrogenic already adherent interpleural connection into a larger one. A ruptured apical bulla, though without having to ascertain the findings through a lung pathology examination, apart from evidence of an apparent long history of nicotine abuse, may equally have contributed to the air entrance into one hemithorax. Given our presentation of the presumably existence of a buffalo chest in a patient after cardiac surgery, it would be reasonable to speculate that the condition may have been related to the previous median sternotomy, although we have no evidence to support this.

Nonetheless there are well-established symptoms of pneumothorax which should warrant urgent confirmation and conservative or invasive treatment if needed, but these signs may not always be so straight forward in real life. Several reports of diagnostic difficulties and pitfalls had led up to delay or missed diagnosis due to the existence of the missing classic signs. ${ }^{9,10}$

Our experience with the presented case calls for a heightened awareness in poststernotomy patients who are presenting with progressive diffuse thoracic pains coupled with respiratory distress and confusion. The elimination of thoracic pain sensation at first with peripheral analgetics and later using central analgetics in our patient may have potentially further masked the symptoms preceding the deterioration. A closer and much earlier reevaluation through chest X-ray or even chest CT-scan in our case may potentially have led to an earlier detection of the pathology.

Needle aspiration which is a well-established method of treatment on suspecting pneumothoraces in the emergency setting may provide a vital clue before body image is available. This should be followed immediately by chest tube drainage after a definite diagnosis is made. ${ }^{5,11}$

\section{References}

1 Barton ED. Tension pneumothorax. Curr Opin Pulm Med 1999;5 (4):269-274

2 Grathwohl KW, Derdak S. Images in clinical medicine. Buffalo chest. N Engl J Med 2003;349(19):1829

3 Schorlemmer GR, Khouri RK, Murray GF, Johnson G Jr. Bilateral pneumothoraces secondary to latrogenic buffalo chest. An unusual complication of median sternotomy and subclavian vein catheterization. Ann Surg 1984;199(3):372-374

4 Hartin DJ, Kendall R, Boyle AA, Atkinson PR. Case of the month: Buffalo chest: a case of bilateral pneumothoraces due to pleuropleural communication. Emerg Med J 2006;23(6):483-486

5 Luh SP. Review: Diagnosis and treatment of primary spontaneous pneumothorax. J Zhejiang Univ Sci B 2010;11(10):735-744

6 Leigh-Smith S, Harris T. Tension pneumothorax-time for a rethink? Emerg Med J 2005;22(1):8-16

7 Saks MA, Griswold-Theodorson S, Shinaishin F, Demangone D. Subacute tension hemopneumothorax with novel electrocardiogram findings. West J Emerg Med 2010;11(1):86-89

8 Shoaib RF, Anwar F, Nyawo B, Sarkar P. Bilateral tension pneumothoraces following coronary artery bypass grafting. J Coll Physicians Surg Pak 2009;19(7):444-446

9 Leigh-Smith S, Davies G. Tension pneumothorax: eyes may be more diagnostic than ears. Emerg Med J 2003;20(5):495-496

10 Watts BL, Howell MA. Tension pneumothorax: a difficult diagnosis. Emerg Med J 2001;18(4):319-320

11 Chan JW, Ko FW, $\mathrm{Ng} \mathrm{CK}$, et al. Management of patients admitted with pneumothorax: a multi-centre study of the practice and outcomes in Hong Kong. Hong Kong Med J 2009;15(6):427-433 